\title{
DIAGNÓSTICO DA VARIABILIDADE ESPACIAL E MANEJO DA FERTILIDADE DO SOLO NO CERRADO
}

\section{Diagnosis and management of spatial variability of soil fertility in the Cerrado}

\author{
Jefferson Santana da Silva Carneiro ${ }^{1}$, Álvaro José Gomes de Faria ${ }^{2}$, Rodrigo Ribeiro Fidelis ${ }^{3}$, Sabino Pereira da Silva \\ $\mathrm{Neto}^{4}$, Antonio Clementino dos Santos ${ }^{5}$, Rubens Ribeiro da Silva ${ }^{6}$
1 Pós Graduando em Ciência do Solo; Departamento de Ciência do Solo; Universidade Federal de Lavras - UFLA; E-mail: carneirojss@yahoo.com.br
${ }_{2}^{2}$ Pós Graduando em Produção Vegetal; Fundação Universidade Federal do Tocantins, UFT, Gurupi - TO; E-mail: ajgomesdefaria@hotmail.com
${ }^{3}$ Professor Adjunto IV; Doutor em Fitotecnia; Fundação Universidade Federal do Tocantins, UFT, Gurupi - TO. E-mail: fidelisrr@uft.edu.br
${ }^{4}$ Professor Doutor em Ciência Animal Tropical; Instituto Federal de Educação, Ciência e Tecnologia do Tocantins (IFTO), Gurupi, TO; E-mail: sabino.pereira@ifto.edu.br Araguaína - TO; E-mail: clementino@uft.edu.br
${ }^{6}$ Professor Adjunto da UFT; Doutor em Solos e Nutrição de Plantas; Fundação Universidade Federal do Tocantins, UFT, Gurupi - TO; E-mail: rrs2002@uft.edu.br \\ ${ }_{5}^{5}$ Professor Associado II; Doutor em Tecnologias Energéticas Nucleares; Fundação Universidade Federal do Tocantins, UFT,
}

Artigo enviado em 08/01/2016, aceito em 19/02/2017 e publicado em 03/03/2017

Resumo - O conhecimento da variabilidade espacial dos atributos químicos do solo permite um manejo mais eficiente da fertilidade. Objetivou-se caracterizar a variabilidade espacial dos atributos químicos e realizar o manejo da fertilidade de um Latossolo Vermelho distrófico no cerrado piauiense por meio das ferramentas da geoestatística. Para a realização do estudo foram retiradas 100 amostras de solo na camada de 0,00-0,20 m, em grid de 100 x $30 \mathrm{~m}$. Foram avaliados o $\mathrm{pH}$, fósforo, potássio, cálcio, magnésio, alumínio trocável, acidez potencial, capacidade de troca de cátions total, capacidade de trocas de cátions efetiva, soma de bases, saturação por alumínio e saturação por bases. Todas as variáveis apresentaram dependência espacial, indicando que o número de amostra foi suficiente para mapear a área em estudo, utilizando-se técnicas da geoestatística. O uso da geoestatística e a elaboração dos mapas de isolinhas por krigagem permitem a visualização das zonas de manejo da fertilidade do solo onde ocorrem os maiores e menores níveis dos atributos químicos avaliados, além da elaboração dos mapas diagnóstico da fertilidade e da recomendação de fertilizantes e corretivos. A geoestatística pode ser usada como ferramenta para estimar os indicadores da fertilidade do solo, possibilitando orientar o manejo da fertilidade para áreas específicas.

Palavras-Chave - agricultura de precisão, geoestatística, krigagem.

\begin{abstract}
The study of the spatial variability of soil chemical attributes allows for more efficient management of fertility. This study aimed to characterize the spatial variability of chemical attributes and perform the management of fertility of an Latosol Red dystrophic in Piauí Savanna through geostatistical tools. For the study were taken from 100 soil samples at a depth of 0.00 to $0.20 \mathrm{~m}$, in grid of $100 \times 30 \mathrm{~m}$. We evaluated the $\mathrm{pH}$, phosphorus, potassium, calcium, magnesium, aluminum exchangeable acidity potential, capacity of total cation, exchange capacity of effective cation exchange, sum of bases, aluminum saturation and base saturation. All variables spatial correlation, indicating the number of sample sufficient to map the area under study, using geostatistical techniques. The use of geostatistics and the preparation of contour maps by kriging allow viewing of management areas of soil fertility where there are the highest and lowest levels of this chemical attributes, in addition to the development of diagnostic maps of fertility and recommendation concerning fertilizers. The geostatistics can be used as a tool to estimate soil fertility indicators, allowing guide the management of fertility for specific areas.
\end{abstract}

Keywords - precision agriculture, geostatistics, kriging. 


\section{INTRODUÇÃO}

A expansão da fronteira agrícola para o cerrado do nordeste brasileiro incorporou ao sistema produtivo solos de baixa fertilidade natural (RESENDE et al., 2014; CARNEIRO et al., 2016), ácidos e pobres em cátions básicos, caracterizado principalmente pela baixa disponibilidade de fósforo e reserva insuficiente de potássio (SILVA, 2013; BOTTEGA et al., 2013), dificultando a produção agrícola.

A produção agropecuária é influenciada, dentre outros fatores, pelos atributos do solo, os quais podem ser férteis ou se tornarem férteis através do manejo adequado. Para tanto é necessário conhecer e quantificar a variação das propriedades químicas, uma vez que os mesmos, por serem sistemas dinâmicos e abertos, estão em constantes modificações (DALCHIAVON et al., 2011b; MONTANARI et al., 2015).

A utilização do solo no sistema convencional de cultivo é o principal responsável pela alteração dos atributos químicos originais, principalmente pelo manejo de corretivos e fertilizantes (NEGREIROS NETO et al., 2014). Nesse sistema as áreas cultivadas são consideradas homogêneas, não levando em conta a variabilidade espacial do solo, com uso da mesma quantidade dos fertilizantes e corretivos em toda a área (ZONTA et al., 2014; MATIAS et al., 2015; CARNEIRO et al., 2016).

Atualmente o sistema de agricultura convencional vem sendo substituído pelas técnicas da agricultura de precisão, na qual as dosagens de corretivos e fertilizantes são aplicadas em quantidade variável de acordo com às necessidades específicas de cada local, baseado na caracterização da variabilidade espacial dos atributos químicos do solo (DALCHIAVON et al., 2011a; BOTTEGA et al., 2013).

Os princípios básicos da experimentação agrícola estabelecida por meio do método estatístico clássico consideram que a variabilidade ocorre de forma inteiramente aleatória (ZANÃO JÚNIOR et al., 2010;
ZONTA et al., 2014), não permitindo o entendimento da variabilidade dos atributos do solo espacialmente. Portanto, torna-se necessário o uso de ferramentas da geoestatística para observar a presença de dependência espacial e compreender esta variabilidade, pois a estatística clássica não leva em consideração a distância na qual as amostras foram coletadas no campo (SILVA NETO et al., 2011a; 2011b; CARNEIRO et al., 2016). O método geoestatístico estuda e modela a estrutura de dependência espacial por meio do ajuste de semivariogramas experimentais, além de permitir os respectivos mapeamentos da área estudada por meio da krigagem que permite interpolar valores em qualquer posição no campo de estudo, sem tendência e com variância mínima (DALCHIAVON et al., 2012; RESENDE et al., 2014).

Para o aprimoramento das práticas agrícolas de manejo do solo e das culturas é necessário conhecer a variabilidade dos atributos químicos no solo por meio de estudos geoestatísticos e adotar práticas de agricultura de precisão, realizando o gerenciamento pontual da qualidade do solo. Com base no exposto, objetivou-se caracterizar a variabilidade espacial dos atributos químicos e o manejo da fertilidade de um Latossolo Vermelho distrófico no cerrado do Piauí por meio das ferramentas geoestatítiscas.

\section{MATERIAL E MÉTODOS}

A pesquisa foi realizada na Fazenda São Pedro do grupo CPM Agrícola na safra 2014/2015. A propriedade está localizada no município de Gilbués no estado do Piauí (Figura 1a), cujas coordenadas geográficas de referência são $09^{\circ} 12^{\prime} 36,36$ " S e $45^{\circ} 21^{\prime} 34,10^{\prime \prime} \mathrm{W}$, com altitude média de $574 \mathrm{~m}$. O clima da região é do tipo Aw (Clima Tropical Chuvoso), com temperatura média de $26,5^{\circ} \mathrm{C}$ e precipitação anual de $1200 \mathrm{~mm}$, com estação chuvosa nos meses de outubro a abril (LEITE et al., 2015). 
(a)
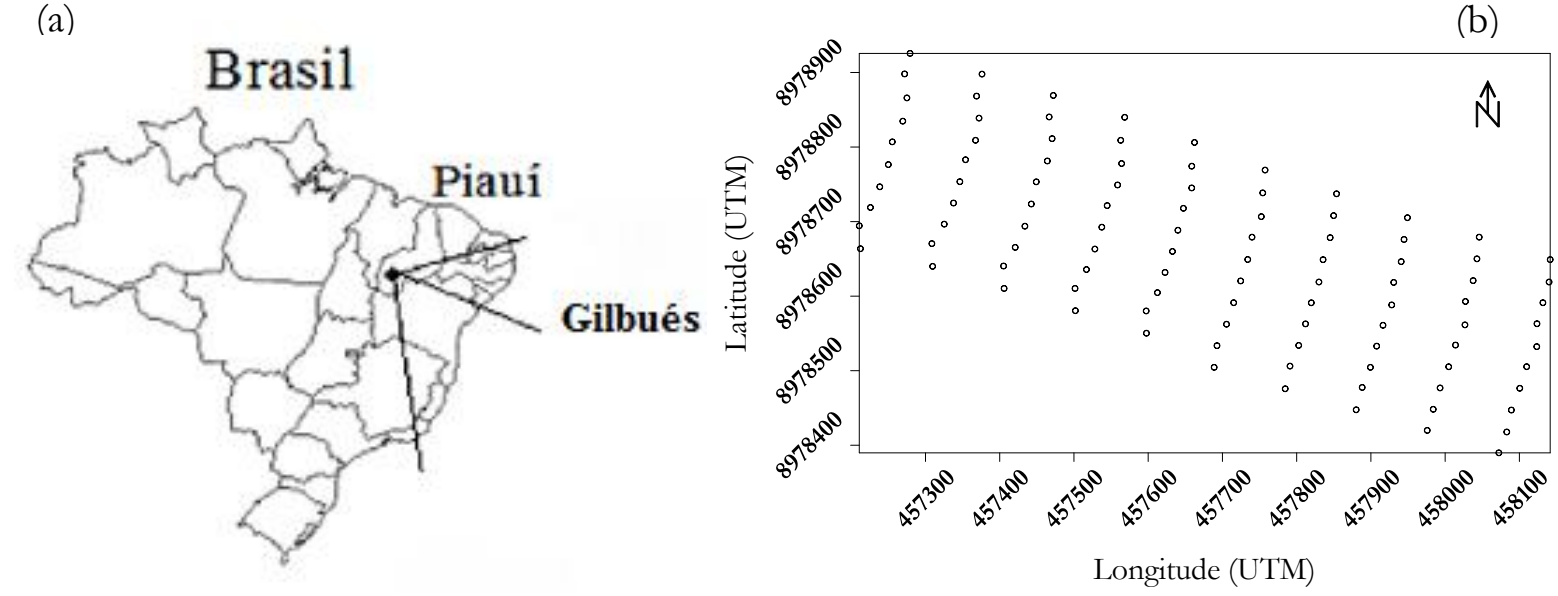

Figura 1: (a) localização da área, Gilbués-Piauí, (b) grid amostral da área recém desmatada e sem cultivo agrícola com 100 pontos em malha de $100 \mathrm{~m}$ x $30 \mathrm{~m}$ em uma área de 24,31 hectares.

O estudo foi realizado em uma área de cultivo comercial da Fazenda São Pedro. A Área foi desmatada no ano de 2014 e não havia sido cultivada, com intervenção apenas na correção do solo com aplicação de 9 toneladas de calcário. O solo estudado foi classificado como Latossolo Vermelho distrófico de textura argilosa (EMBRAPA, 2013), com teores médios de $368,8 \mathrm{~g} \mathrm{~kg}^{-1}$ de areia, 78,6 $\mathrm{g} \mathrm{kg}^{-1}$ de silte e 552,6 $\mathrm{g} \mathrm{kg}^{-1}$ de argila.

As amostras de solo foram coletadas na camada de $0,00-0,20 \mathrm{~m}$, em malha regular de $100 \mathrm{~m}$ × $30 \mathrm{~m}$ (Figura 1b) em 24,31 hectares com pontos distanciados perpendicularmente entre si. No total foram coletadas 100 amostras na área estudada. Cada ponto coletado foi georreferenciado, como forma de demarcar a área de estudo e elaborar os mapas de fertilidade do solo por meio de ferramentas geoestatísticas.

$\mathrm{Na}$ avaliação das características químicas do solo foram determinados: $\mathrm{pH}$ em $\mathrm{H}_{2} \mathrm{O}, \mathrm{P}$ e $\mathrm{K}^{+}$disponíveis (Mehlich $\left.{ }^{-1}\right), \mathrm{Ca}^{2+}, \mathrm{Mg}^{2+} \mathrm{e} \mathrm{Al}{ }^{3+}$ trocáveis $\left(\mathrm{KCl} 1 \mathrm{~mol} \mathrm{~L}^{-1}\right)$ e acidez potencial $(\mathrm{H}+\mathrm{Al})\left(\mathrm{Ca}(\mathrm{OAc}) 20,5 \mathrm{~mol} \mathrm{~L}^{-1}\right)$, (EMBRAPA, 2011). Com os valores obtidos nas análises do solo, calculou-se a capacidade de trocas de cátions total e efetiva $\left(\mathrm{CTC}_{(\mathrm{T})}\right.$ e CTCe) a pH 7,0, soma de bases (SB), porcentagem de saturação por alumínio $(\mathrm{m} \%)$ e porcentagem de saturação por bases (V\%).

Para a análise estatística dos dados, inicialmente realizou-se estudo exploratório, calculou-se medidas de localização (média, mediana, mínimo e máximo), de variabilidade (coeficiente de variação) e de tendência central (assimetria e curtose) para verificar a normalidade dos atributos avaliados. A hipótese de normalidade dos dados foi verificada com o teste de Shapiro \& Wilk (1965) a nível de 5\% de probabilidade. Para a análise do coeficiente de variação (CV), usou-se a classificação de Warrick \& Nielsen (1980), com variabilidade baixa para valores $<12 \%$, média para valores entre $12 \%<\mathrm{CV}<60 \%$ e alta para valores $>$ $60 \%$.

Para determinar a variabilidade espacial considerou-se a teoria das variáveis regionalizadas, a qual dispõe de diferentes métodos de análise de variação espacial, e uma delas é o semivariograma. Os semivariogramas de cada atributo foram obtidos e ajustados aos modelos experimentais linear, esférico, exponencial e gaussiano. Por meio destes modelos foi realizada a predição de cada atributo em zonas não amostradas por meio de interpolações mediante o uso da krigagem, representados em mapas de contorno.

Foi realizado ainda o mapeamento do diagnóstico da fertilidade do solo e posteriormente os mapas de recomendação de corretivos e fertilizantes segundo Ribeiro et al. (1999) para a cultura da soja e em seguida realizado o levantamento de custos com a correção e a fertilização da área.

A escolha dos modelos teóricos de semivariogramas foi efetivada observando-se a menor soma do quadrado dos resíduos (SQR) e o maior coeficiente de determinação $\left(\mathrm{R}^{2}\right)$. O ajuste do modelo de semivariograma escalonado possibilitou definir os seguintes parâmetros: efeito pepita $\left(\mathrm{C}_{0}\right)$, patamar $\left(C_{0}+C_{1}\right)$, alcance $\left(A_{0}\right)$ e o grau de dependência espacial (GDE).

A classificação do grau da dependência espacial (GDE) foi feita com base na razão entre o efeito pepita e o patamar conforme a equação 1 .

$\mathrm{GDE}=\left[\mathrm{C}_{1} /\left(\mathrm{C}_{0}+\mathrm{C}_{1}\right)\right]^{* 100}$

(Equação 1)

Onde: $\mathrm{GDE}=$ grau de dependência espacial; $\mathrm{C}_{0}=$ efeito pepita; $\mathrm{C}_{1}=$ variância estrutural; $\mathrm{C}_{0}+\mathrm{C}_{1}=$ patamar.

A interpretação do grau de dependência espacial para escolha do modelo foi de acordo com a seguinte 
classificação: GDE < 20\%, considerada dependência espacial muito baixa; $20 \% \leq$ GDE $<40 \%$, considerada dependência espacial baixa; $40 \% \leq$ GDE $<60 \%$, considerada dependência espacial média; $60 \% \leq \mathrm{GDE}$ $<80 \%$, considerada dependência espacial alta; $80 \% \leq$ GDE $<100 \%$, considerada dependência espacial muito alta (DALCHIAVON et al., 2012).

\section{RESULTADOS E DISCUSSÃO}

A análise estatística descritiva dos valores dos atributos químicos do Latossolo Vermelho distrófico possibilitou verificar que, os valores médios apresentaram-se abaixo do nível crítico considerado para o bom desenvolvimento da maioria das culturas agrícolas (RIBEIRO et al., 1999). De maneira geral, a área pode ser considerada de baixa fertilidade, uma vez que a saturação por bases $(\mathrm{V} \%)$ encontra-se abaixo de $50 \%$. No entanto o solo apresenta valor de capacidade de troca catiônica total $\left(\mathrm{CTC}_{(\mathrm{T})}\right)$ elevado, assim a saturação por bases verificada é suficiente para garantir o bom desenvolvimento das plantas.

A $\quad \mathrm{CTC}_{(\mathrm{T})}$ corresponde à soma das cargas negativas nas partículas microscópicas do solo retendo os cátions, tais como $\mathrm{Ca}^{2+}, \mathrm{Mg}^{2+}, \mathrm{K}^{+}, \mathrm{Na}^{+}, \mathrm{Al}^{3+}$ e $\mathrm{H}^{+}$. A $\mathrm{CTC}_{(\mathrm{T})}$ é de grande importância no que diz respeito à fertilidade do solo, uma vez que indica a capacidade total de retenção de cátions, os quais, em geral, irão tornar-se disponíveis às plantas, assim mesmo estando a $\mathrm{V} \%$ abaixo de $50 \%$ a quantidade de cátions retida está acima do mínimo recomendado para a maioria das culturas agrícolas.

Os valores de média e mediana da maioria dos atributos químicos do Latossolo Vermelho estão próximos, evidenciando que estes apresentam ou se aproximam da distribuição normal. A maioria dos atributos químicos avaliados apresentaram distribuição normal. A ausência de normalidade em alguns dos atributos químicos do solo deve-se, provavelmente à variação natural que ocorre no processo de formação do solo. Daí a importância de se adotar métodos com maior precisão no manejo da fertilidade do solo, tratando de forma pontual cada necessidade do solo.

A variabilidade dos atributos químicos pode ser classificada em função do seu coeficiente de variação (CV). O solo apresentou CV baixo $(\mathrm{CV}<12 \%)$ para $\mathrm{pH}$ e CVs médios $(12 \%<\mathrm{CV}<60 \%)$ para os demais atributos químicos do solo (Tabela 1). As variações dos atributos químicos do solo são consequências de complexas interações dos processos de sua formação e de práticas de manejo do solo da cultura, provocadas pelas adubações e calagens sucessivas e irregulares com impacto principalmente nas camadas superficiais do solo (BOTTEGA et al., 2013; CARNEIRO et al., 2016).

Tabela 1- Estatística descritiva dos atributos químicos do Latossolo Vermelho distrófico no cerrado Piauiense, Gilbués, Piauí (2016)

\begin{tabular}{|c|c|c|c|c|c|c|c|c|}
\hline \multirow{2}{*}{ Atributo Químico } & \multirow{2}{*}{ Média } & \multirow{2}{*}{ Mediana } & \multicolumn{2}{|c|}{ Valor } & \multicolumn{3}{|c|}{ Coeficientes } & \multirow{2}{*}{ Teste $\mathrm{W}^{(1)}$} \\
\hline & & & Mínimo & Máximo & $\mathrm{CV}^{(2)}$ & $\mathrm{Cs}^{(3)}$ & $\mathrm{Ck}^{(4)}$ & \\
\hline $\mathrm{pH} \mathrm{H}{ }_{2} \mathrm{O}$ & 4,390 & 4,350 & 3,900 & 5,500 & 6,528 & 1,252 & 3,082 & $0,918^{\mathrm{ns}}$ \\
\hline $\mathrm{P}\left(\mathrm{mg} \mathrm{dm}^{-3}\right)$ & 2,823 & 2,606 & 1,247 & 5,080 & 30,394 & 0,332 & $-0,820$ & $0,965^{\mathrm{ns}}$ \\
\hline $\mathrm{K}^{+}\left(\mathrm{mg} \mathrm{dm}^{-3}\right)$ & 35,201 & 33,373 & 13,669 & 83,174 & 36,215 & 0,956 & 1,545 & $0,950^{\mathrm{ns}}$ \\
\hline $\mathrm{Ca}^{2+}\left(\mathrm{cmol}_{\mathrm{c}} \mathrm{dm}^{-3}\right)$ & 2,043 & 1,910 & 0,700 & 4,100 & 37,548 & 0,489 & $-0,380$ & $0,971^{\mathrm{ns}}$ \\
\hline $\mathrm{Mg}^{2+}\left(\mathrm{cmol}_{\mathrm{c}} \mathrm{dm}^{-3}\right)$ & 1,784 & 1,708 & 0,225 & 3,650 & 42,682 & 0,333 & $-0,182$ & $0,981 *$ \\
\hline $\mathrm{Al}^{3+}\left(\mathrm{cmol}_{\mathrm{c}} \mathrm{dm}^{-3}\right)$ & 1,543 & 1,505 & 0,120 & 3,000 & 38,478 & 0,121 & $-0,006$ & $0,991 *$ \\
\hline $\mathrm{H}+\mathrm{Al}\left(\mathrm{cmol}_{\mathrm{c}} \mathrm{dm}^{-3}\right)$ & 12,760 & 12,738 & 5,585 & 21,953 & 20,603 & 0,122 & 1,341 & $0,982 *$ \\
\hline $\mathrm{SB}\left(\mathrm{cmol}_{\mathrm{c}} \mathrm{dm}^{-3}\right)$ & 3,931 & 3,859 & 1,709 & 7,191 & 29,207 & 0,379 & $-0,198$ & $0,983^{*}$ \\
\hline $\mathrm{CTC}_{(\mathrm{T})}\left(\mathrm{cmol}_{\mathrm{c}} \mathrm{dm}^{-3}\right)$ & 16,678 & 16,582 & 11,121 & 25,319 & 15,373 & 0,523 & 1,024 & $0,975^{*}$ \\
\hline CTCe $\left(\mathrm{cmol}_{\mathrm{c}} \mathrm{dm}^{-3}\right)$ & 5,472 & 5,500 & 3,566 & 7,911 & 16,650 & 0,265 & $-0,217$ & $0,990^{*}$ \\
\hline V $(\%)$ & 23,855 & 22,536 & 11,679 & 49,779 & 33,272 & 0,896 & 0,702 & $0,946^{\mathrm{ns}}$ \\
\hline $\mathrm{m}(\%)$ & 29,411 & 29,050 & 2,113 & 62,105 & 42,385 & 0,062 & $-0,390$ & $0,993 *$ \\
\hline
\end{tabular}

P: fósforo; $\mathrm{K}^{+}$: potássio; $\mathrm{Ca}^{2+}$ : cálcio; $\mathrm{Mg}^{2+}$ : magnésio; $\mathrm{Al}^{3+}$ : alumínio trocável; $\mathrm{H}+\mathrm{Al}$ : acidez potencial; $\mathrm{SB}$ : soma de bases; $\mathrm{CTC}_{(\mathrm{T})}$ : capacidade de troca catiônica total; CTCe: capacidade de troca catiônica efetiva; V\%: saturação por bases; m\%: saturação por alumínio. (1)W: teste de normalidade de Shapiro \& Wilk; (2) CV: coeficiente de variação; ${ }^{(3)} \mathrm{Cs}$ : coeficiente de assimetria; (4)Ck: coeficiente de curtose; ${ }^{(*)}$ significativo ao teste de normalidade de Shapiro \& Wilk à $5 \%$ de probabilidade; (ns) não significativo ao teste de normalidade de Shapiro \& Wilk à $5 \%$ de probabilidade.

Os atributos químicos do solo apresentaram distribuição assimétrica positiva, sendo os maior valor observado para $\mathrm{pH}(1,250)$ e o menor para o $\mathrm{m} \%$ $(0,062)$. O coeficiente de assimetria (Cs) é utilizado para caracterizar como e quanto a distribuição de frequências se afasta da simetria. Se o valor encontrado para esse coeficiente for zero, a distribuição é simétrica; se positivo, a distribuição é assimétrica à direita; e se negativo, é assimétrica à esquerda (ZANÃO JUNIOR et al., 2010).

Os coeficientes de curtose também podem ser utilizados para avaliar se os dados seguem distribuição 
normal, que deve ser preferencialmente nulos e pode ser aceitos valores entre $+2 \mathrm{e}-2$ (NEGREIROS NETO et al. (2014). Observa-se na Tabela 1 que os valores de coeficientes de curtose $(\mathrm{Ck})$ dos atributos avaliados apresentaram-se dentro dos limites considerados como aceitáveis $(-2<\mathrm{Ck}<+2)$, exceto os atributos $\mathrm{pH}(3,082)$ que não se apresentou nos limites indicados por Negreiros Neto et al. (2014).

Os parâmetros de ajuste dos semivariogramas foram estimados pelas ferramentas geoestatísticas para avaliar a dependência espacial dos atributos e os alcances da autocorrelação espacial, foi comparada para os diferentes atributos avaliados.

No estudo dos semivariogramas o alcance é parâmetro importante, que indica a distância máxima em que a variável está correlacionada espacialmente, delimitando a extensão da correlação espacial entre as amostras (DALCHIAVON et al., 2012; CARNEIRO et al., 2016). As determinações realizadas a distâncias maiores que o alcance têm distribuição aleatória e, por essa razão, são independentes entre si, devendo ser aplicada a estatística clássica (MATIAS et al., 2015). Por outro lado, determinações realizadas em distâncias menores que o alcance, são correlacionadas, o que permite que se façam interpolações para espaçamentos menores que os amostrados (NEGREIROS NETO et al., 2014).

Diferentes valores de alcance foram encontrados para os atributos químicos avaliados. $\mathrm{Na}$ Tabela 2 podese observar que os valores do alcance obtidos variaram de 7,92 $\left(\mathrm{H}+\mathrm{Al}, \mathrm{CTC}_{(\mathrm{T})}\right)$ a $1955,31 \mathrm{~m}\left(\mathrm{Al}^{3+}\right)$, os quais correspondem aos raios das áreas consideradas homogêneas para cada variável estudada (LIMA et al., 2014; RESENDE et al., 2014).

Tabela 2: Componentes da semivariância e Grau de Dependência Espacial (GDE) dos atributos químicos do Latossolo Vermelho distrófico no cerrado Piauiense, Gilbués, Piauí (2016)

\begin{tabular}{|c|c|c|c|c|c|c|c|c|}
\hline \multirow{2}{*}{$\begin{array}{l}\text { Atributo } \\
\text { Químico }\end{array}$} & \multicolumn{3}{|c|}{ Semivariância } & \multirow{2}{*}{$\begin{array}{c}\text { Alcance } \\
\left(\mathrm{A}_{0}\right)\end{array}$} & \multirow{2}{*}{$\mathrm{R}^{2(2)}$} & \multirow{2}{*}{ Modelo $^{(3)}$} & \multirow{2}{*}{$\begin{array}{c}\operatorname{GDE}^{(1)} \\
(\%)\end{array}$} & \multirow{2}{*}{ Classe* } \\
\hline & $\left(\mathrm{C}_{0}\right)^{(\mathrm{a})}$ & $\left(\mathrm{C}_{1}\right)^{(\mathrm{b})}$ & $\left(\mathrm{C}_{0}+\mathrm{C}_{1}\right)^{(\mathrm{c})}$ & & & & & \\
\hline $\mathrm{pH} \mathrm{H}{ }_{2} \mathrm{O}$ & 0,0229 & 0,0879 & 0,1108 & 177,10 & 0,631 & Exp. & 79,33 & AL \\
\hline $\mathrm{P}(4)$ & 0,1078 & 0,6352 & 0,7430 & 39,10 & 0,663 & Gau. & 85,49 & MA \\
\hline $\mathrm{K}^{+(4)}$ & 72,000 & 134,90 & 206,90 & 168,20 & 0,725 & Gau. & 65,20 & AL \\
\hline $\mathrm{Ca}^{2+(5)}$ & 0,0010 & 0,5690 & 0,5700 & 8,71 & 0,933 & Gau. & 99,83 & MA \\
\hline $\mathrm{Mg}^{2+(5)}$ & 0,2280 & 0,3940 & 0,6220 & 260,60 & 0,738 & Esf. & 63,34 & AL \\
\hline $\mathrm{Al}^{3+(5)}$ & 0,2460 & 0,1423 & 0,3883 & 1955,31 & 0,716 & Gau. & 36,65 & $\mathrm{BA}$ \\
\hline $\mathrm{H}+\mathrm{Al}^{(5)}$ & 0,0100 & 6,6770 & 6,6870 & 7,92 & 0,864 & Gau. & 99,85 & MA \\
\hline $\mathrm{SB}^{(5)}$ & 0,0010 & 1,2880 & 1,2890 & 8,71 & 0,881 & Gau. & 99,92 & MA \\
\hline $\mathrm{CTC}_{(\mathrm{T})}{ }^{(5)}$ & 0,0100 & 6,226 & 6,2360 & 7,92 & 0,811 & Gau. & 99,84 & MA \\
\hline $\mathrm{CTC}_{\mathrm{e}}{ }^{(5)}$ & 0,1010 & 0,7030 & 0,8040 & 10,54 & 0,779 & Gau. & 87,44 & MA \\
\hline V $(\%)$ & 0,1000 & 61,980 & 62,080 & 8,71 & 0,951 & Gau. & 99,83 & MA \\
\hline $\mathrm{m}(\%)$ & 51,000 & 133,30 & 184,30 & 143,30 & 0,645 & Exp. & 72,33 & $\mathrm{AL}$ \\
\hline
\end{tabular}

P: fósforo; $\mathrm{K}^{+}$: potássio; $\mathrm{Ca}^{2+}$ : cálcio; $\mathrm{Mg}^{2+}$ : magnésio; $\mathrm{Al}^{3+}$ : alumínio trocável; $\mathrm{H}+\mathrm{Al}$ : acidez potencial; $\mathrm{SB}$ : soma de bases; $\mathrm{CTC}_{\mathrm{T}}$ : capacidade de troca catiônica total; CTCe: capacidade de troca catiônica efetiva; $\mathrm{V} \%$ : saturação por bases; $\mathrm{m} \%$ : saturação por alumínio. ${ }^{(1)}$ GDE: Grau de dependência espacial - $\left(\mathrm{C}_{1} /\left(\mathrm{C}_{0}+\mathrm{C}_{1}\right)\right) * 100$; *Classes: MB: Muito Baixo; BA: Baixo; ME: Médio; AL: Alto; MA: Muito Alto; (2) $\mathrm{R}^{2}$ : coeficiente de determinação; (3)Modelos experimentais: Esf: esférico; Gau: gaussiano; Exp: exponencial; Epp: efeito pepita puro. ${ }^{(4)}$ dados em mg dm${ }^{-3}$; (5) dados em cmol $_{\mathrm{c}} \mathrm{dm}^{-3}$; (a) $\mathrm{C}_{0}$ : Efeito pepita; ${ }^{(b)} \mathrm{C}_{1}$ : Contribuição; ${ }^{(c)} \mathrm{C}_{0}+\mathrm{C}_{1}$ : patamar.

Os valores de alcance são influenciados pelas práticas agronômicas, principalmente pela aplicação de corretivos e fertilizantes, uma vez que o objetivo é realizar o homogeneização da área de cultivo. Quanto mais homogênea é a área de cultivo, maior será o raio de correlação espacial dos atributos avaliados, ou seja, maior será o alcance. A adoção da agricultura de precisão tratando de forma pontual cada necessidade tende a uniformizar a fertilidade do solo, diminuindo da variabilidade espacial e aumentando o alcance geoestatístico.

O alcance de grande parte dos atributos, apresentou-se inferior a $10 \mathrm{~m}$. Esses atributos possuem pouca continuidade (correlação entres os pontos amostrados), ou seja, dependência espacial em raio muito pequeno (Tabela 2). Tal característica pode ser explicada pela pouca interferência antrópica na área, uma vez que a área não foi cultivada, apresentando na sua maioria variabilidade espacial influenciada pelas características de formação do solo e pela deposição de materiais da vegetação anterior ao desmatamento.

$\mathrm{O}$ modelo que mais se adequou aos semivariogramas experimentais dos atributos químicos do solo avaliados foi o gaussiano, exceto para saturação por alumínio $(\mathrm{m} \%)$ e $\mathrm{pH}$ que apresentaram melhor ajuste ao modelo exponencial, e magnésio $\left(\mathrm{Mg}^{2+}\right)$ que se ajustou ao modelo esférico.

A principal diferença entre o modelo exponencial e o esférico é que o exponencial atinge o patamar apenas assintoticamente, enquanto que o modelo esférico o atinge no valor do alcance (DRUCK et al., 2004). Os parâmetros $\left(C_{0}\right)$ e $\left(C_{0}+C_{1}\right)$ para o modelo exponencial são determinados da mesma maneira que para o esférico. $\mathrm{O}$ atributo que apresentou melhor ajuste ao semivariograma foi a saturação por 
bases $(\mathrm{V} \%)$ com coeficiente de determinação $\left(\mathrm{R}^{2}\right)$ de 0,951 .

Efetuada por meio dos semivariogramas experimentais a análise geoestatística permitiu detectar diferentes escalas de variabilidade espacial. Os modelos ajustados pelos semivariogramas nas análises dos atributos químicos do solo apresentaram dependência espacial (Tabela 2), ou seja, a distribuição desses atributos no espaço não é aleatória. As relações entre a contribuição $\left(\mathrm{C}_{1}\right)$ e o patamar $\left(\mathrm{C}_{0}+\mathrm{C}_{1}\right)$ demonstram predominância de grau de dependência espacial (GDE) muito alta seguida de alta.

Para a maioria dos atributos químicos foi observada dependência espacial muito alta e alta, exceto
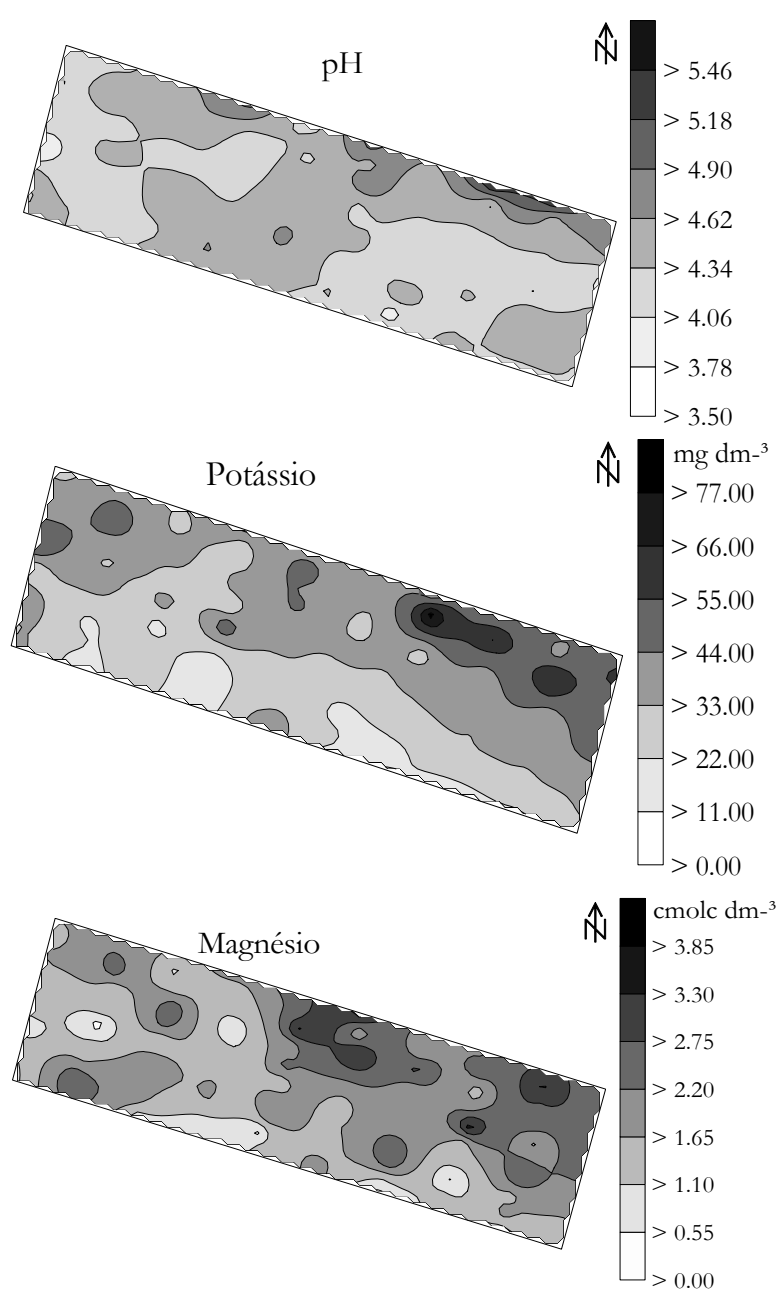

$\mathrm{Al}^{3+}$ classificado como baixo GDE. A variável com forte dependência espacial é quando o efeito pepita for menor ou igual a $25 \%$ do patamar (CAMBARDELLA et al., 1994). Os resultados obtidos das varáveis analisadas evidencia valores próximos aos pontos de amostragem vizinhos do que ao restante das amostragens mais distantes. Com a forte dependência espacial, favoreceu o melhor ajuste dos variogramas, permitindo deste modo a interpolação dos dados.

A partir do ajuste dos semivariogramas, foi possível elaborar os mapas de isolinhas (Figura 2) com a interpolação de valores em qualquer posição no campo em estudo, por krigagem ordinária.
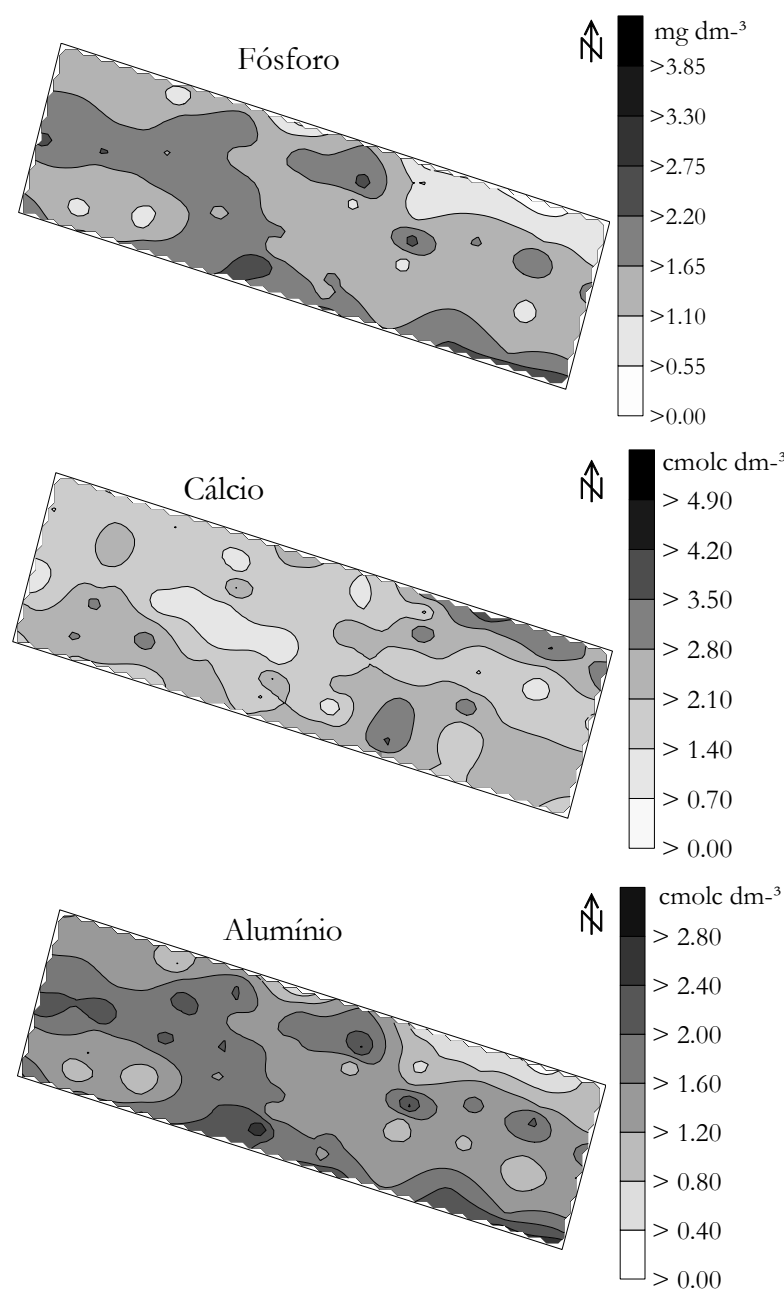

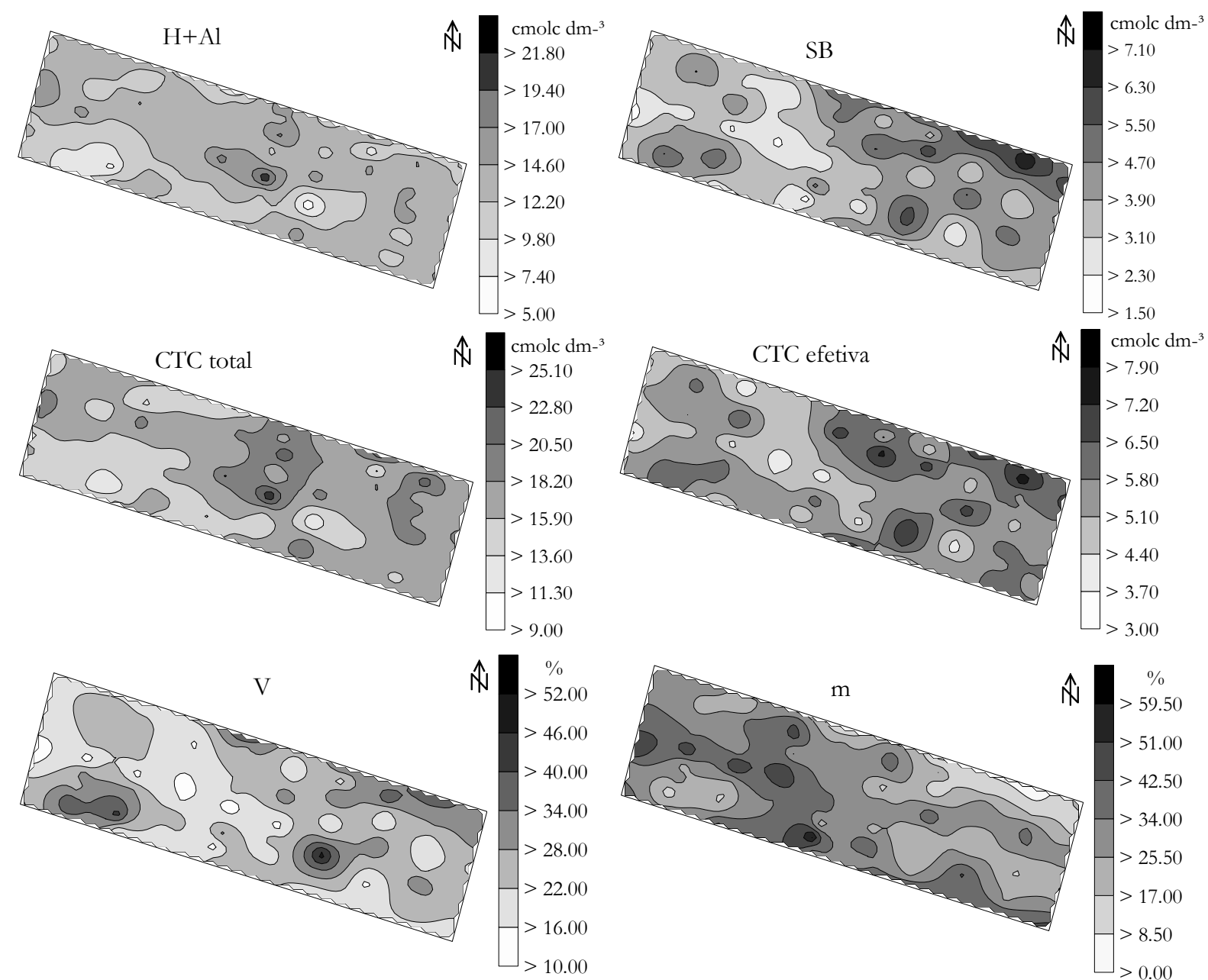

Figura 2: Mapas da distribuição espacial da fertilidade do Latossolo Vermelho distrófico no cerrado Piauiense, Gilbués, Piauí (2016). pH: em água $\left(\mathrm{H}_{2} \mathrm{O}\right)$; $\mathrm{H}+\mathrm{Al}$ : acidez potencial; SB: soma de bases; $\mathrm{CTC}$ total: capacidade de troca catiônica total; CTC efetiva: capacidade de troca catiônica efetiva; V: saturação por bases; m: saturação por alumínio.

Nos mapas (Figura 2) gerados pode-se visualizar a distribuição espacial dos teores dos nutrientes e atributos químicos avaliados. Por meio dos mapas de isolinhas da distribuição dos atributos químicos do solo é possível verificar que houve ampla variação dos atributos na área avaliada, confirmando os resultados de coeficientes de variação (CVs) verificados na análise descritiva da distribuição dos dados. Os mapas de distribuição obtidos por meio da interpolação dos dados, pelo método da krigagem ordinária, são fundamentais na avaliação da fertilidade do solo, o que permite a visualização zonas especificas de manejo, as quais são informações importantes para o aumento da precisão na agricultura, e possibilita a aplicação de insumos com taxas variadas, visando à homogeneização da fertilidade do solo.

Seguindo o método de recomendação da agricultura convencional com base nos valores médios dos atributos químicos (Tabela 1 ) onde a recomendação dos corretivos e fertilizantes são extrapolados para toda área, classificou-se os atributos segundo Ribeiro et al. (1999), como muito alto para acidez potencial $(\mathrm{H}+\mathrm{Al})$, alto para alumínio trocável $\left(\mathrm{Al}^{3+}\right)$, muito bom para capacidade de troca catiônica total $\left(\mathrm{CTC}_{(\mathrm{T})}\right)$, bons ou adequados para cálcio $\left(\mathrm{Ca}^{2+}\right)$, magnésio $\left(\mathrm{Mg}^{2+}\right)$ e soma de bases (SB), médio para capacidade de troca catiônica efetiva (CTCe), baixo para potássio $\left(\mathrm{K}^{+}\right)$, saturação por bases (V\%), saturação por alumínio (m\%) e pH e muito baixo para o fósforo (P).

Uma análise mais detalhada dos mapas de fertilidade permitiu a elaboração dos mapas diagnósticos dos atributos do solo tomando como base a classificação de Ribeiro et al. (1999). Os mapas diagnósticos da fertilidade do solo (Figura 3) foram realizados a partir do mapa da distribuição espacial dos atributos químicos do solo identificando as zonas onde os atributos estão muito baixos, baixo, médio, bom ou muito bom. 

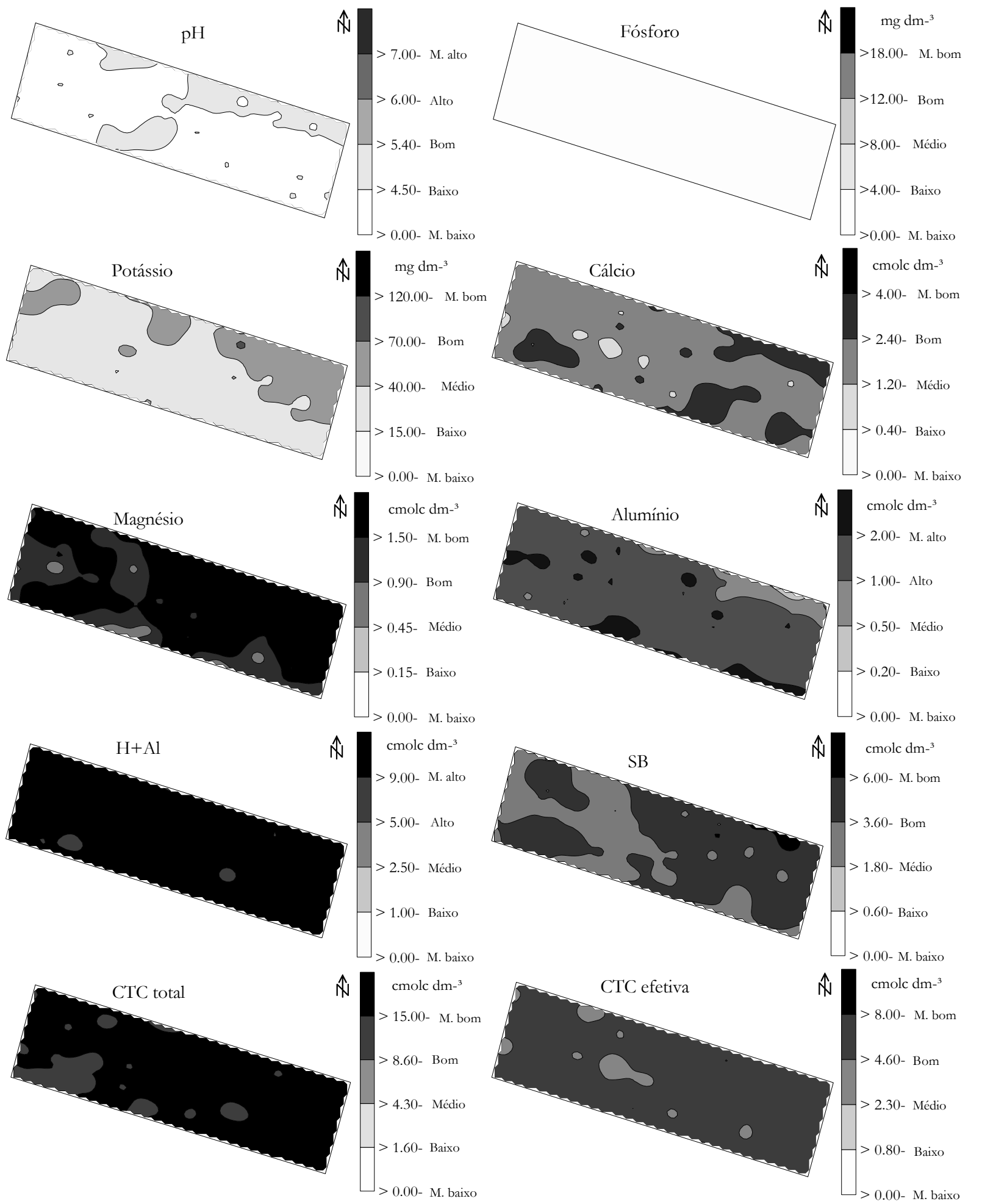

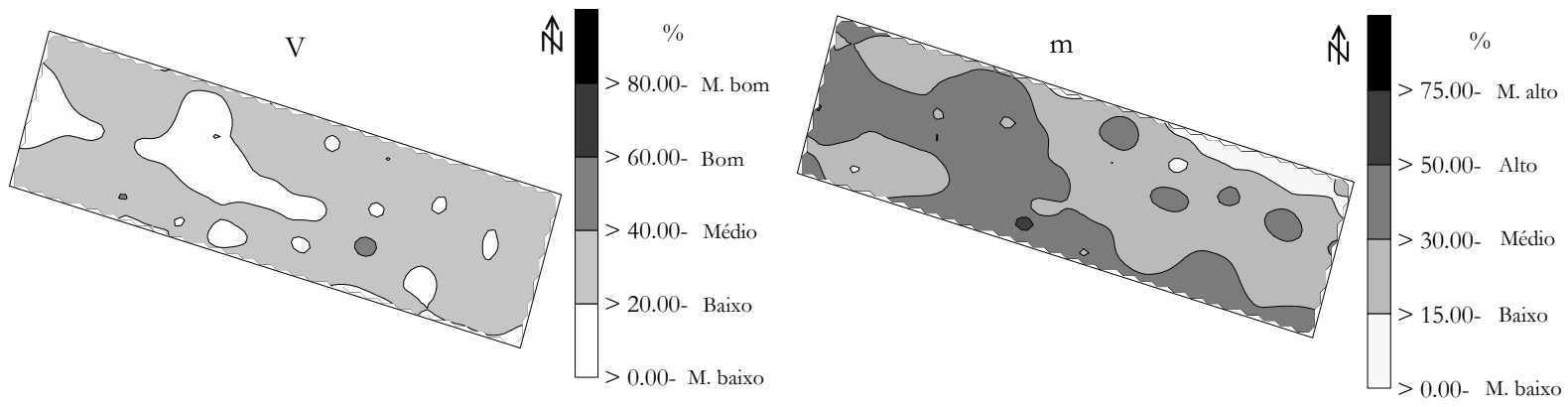

Figura 3: Mapas diagnósticos da fertilidade do Latossolo Vermelho distrófico no cerrado Piauiense, Gilbués, Piauí (2016). pH: em água $\left(\mathrm{H}_{2} \mathrm{O}\right) ; \mathrm{H}+\mathrm{Al}$ : acidez potencial; SB: soma de bases; CTC total: capacidade de troca catiônica total; CTC efetiva: capacidade de troca catiônica efetiva; V: saturação por bases; m: saturação por alumínio.

Os bons resultados observados para cálcio e magnésio, assim como dos atributos influenciados por esses elementos, só foi obtido devido ao fato de ter acorrido aplicação de 9 toneladas de calcário por hectare na área antes da coleta do solo, divididas em três aplicações. Já a baixa disponibilidade de $\mathrm{P}$ e $\mathrm{K}^{+}$ observada é característica natural dos solos do cerrado, em especial os Latossolos sob condição natural de fertilidade os quais apresentam baixa disponibilidade de cátions básicos e nutrientes, principalmente $\mathrm{P}$ (BOTTEGA et al., 2013; SILVA, 2013).

Os mapas da distribuição espacial e diagnóstico da fertilidade do solo (Figuras 2 e 3 ) possibilitam manejo adequado da área e indicam áreas prioritárias de manejo, ou seja, aquelas que possuem deficiências nutricionais que podem vir a impossibilitar o alcance de grandes produções agrícolas. Uma análise visual dos mapas elaborados permitiu identificar as zonas onde os atributos encontram-se dentro da classe considerada como adequada para o cultivo agrícola, além de verificar a variabilidade da fertilidade do solo. A elaboração de mapas de variabilidade espacial e de grande importância para a agricultura de precisão, uma vez que de posse desses mapas é possível realizar um planejamento e manejo de forma homogênea da fertilidade do solo, visando a padronização dos níveis de fertilidade da aérea a ser trabalhada.

Os mapas de recomendação de corretivos e fertilizantes (Figura 4) foram realizados de acordo com as exigências da cultura da soja que seria implantada na safra 2014/2015.

(a)
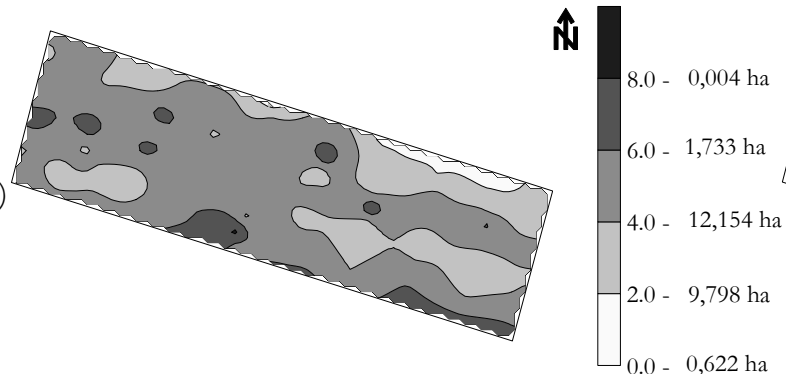

(b)

4,559

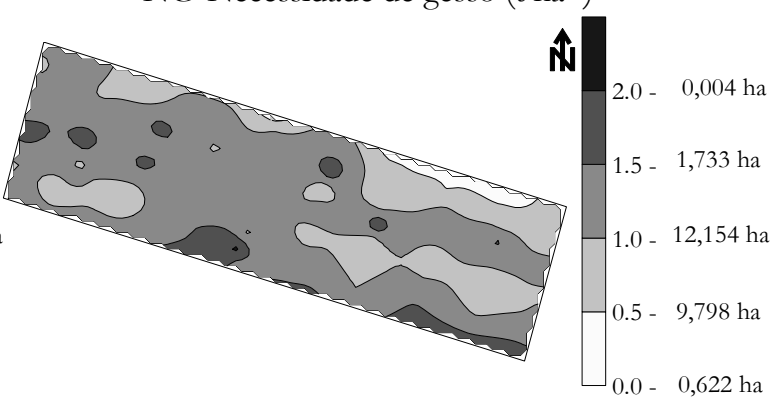

1,140

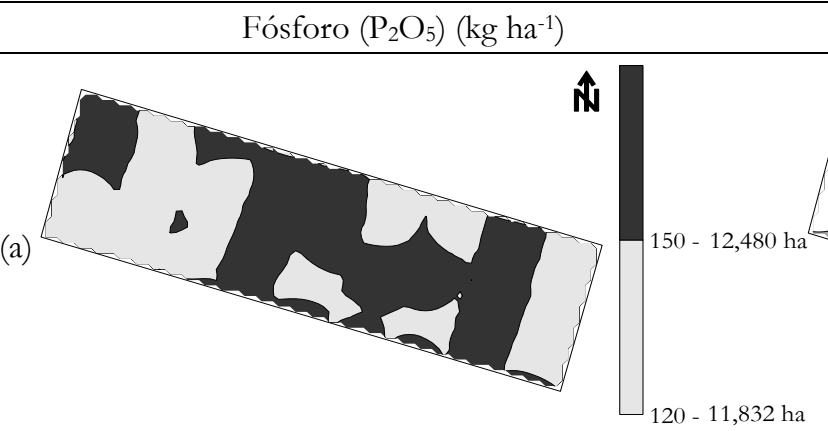

(b)

150

Potássio $\left(\mathrm{K}_{2} \mathrm{O}\right)\left(\mathrm{kg} \mathrm{ha}^{-1}\right)$

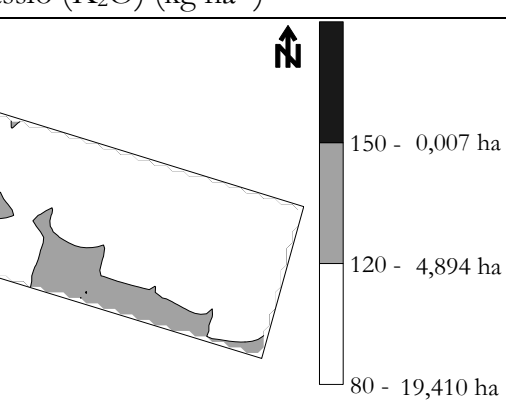

120 
Figura 4: Mapas da variabilidade espacial da recomendação de fertilizantes e corretivos e recomendação pela média da fertilidade do Latossolo Vermelho distrófico no cerrado Piauiense, Gilbués, Piauí (2016). (a) taxa variada; (b) recomendação pela média; Recomendação realizada para a cultura da soja; NC: Necessidade de Calagem (t ha-1); QC: Quantidade de calcário; NG: Necessidade de Gesso $\left(\mathrm{t} \mathrm{ha}^{-1}\right)$; QG: Quantidade de gesso; NC=QC (SC:100\%; PF: 20 cm; PRNT:100); NG=QG (SC:100\%; PF: $20 \mathrm{~cm}$ ).

Os mapas de recomendação de corretivos e fertilizantes (Figura 4) mostram quando realizado a aplicação de forma variável de acordo com as necessidades especificas, ocorrem zonas com maior e menor exigência desses insumos. Daí a importância da adoção da agricultura de precisão no processo de gerenciamento da fertilidade do solo na produção agrícola. A aplicação pontual dos insumos além de homogeneizar a fertilidade do solo permite uma economia com aquisição dos insumos, uma vez que há um melhor controle na distribuição. A tabela 3 mostra o consumo de corretivos e fertilizantes e os custos da aplicação a taxa variada e da aplicação baseada na recomendação média da fertilidade do solo.

Tabela 3-Quantidade de corretivos, fertilizantes e custos para a correção da fertilidade do solo utilizando a aplicação a taxa variada e recomendação pela média geral da fertilidade do solo no cerrado Piauiense, Gilbués, Piauí (2016)

\begin{tabular}{cccccccc}
\hline $\begin{array}{c}\mathrm{NC} \\
(\mathrm{t})\end{array}$ & $\begin{array}{c}\text { Custo com calcário } \\
(\mathrm{R} \$)\end{array}$ & $\begin{array}{c}\mathrm{NG} \\
(\mathrm{t})\end{array}$ & $\begin{array}{c}\text { Custo com gesso } \\
(\mathrm{R} \$)\end{array}$ & $\begin{array}{c}\mathrm{P}_{2} \mathrm{O}_{5}(1) \\
(\mathrm{t})\end{array}$ & $\begin{array}{c}\text { Custo com MAP } \\
(\mathrm{R} \$)\end{array}$ & $\begin{array}{c}\mathrm{K}_{2} \mathrm{O}^{(2)} \\
(\mathrm{t})\end{array}$ & $\begin{array}{c}\text { Custo com } \mathrm{KCl} \\
(\mathrm{R} \$)\end{array}$ \\
\hline 78,642 & 3538,89 & 19,66 & 1061,64 & 3,292 & 5112,48 & 2,141 & 3136,57 \\
\hline \multicolumn{7}{c}{ Taxa variada } \\
\hline 110,83 & 4987,32 & 27,71 & \multicolumn{7}{c}{ Recomendação pela média } \\
\hline
\end{tabular}

Recomendação e custos para área total: 24,31 ha; (1) Fonte de Fertilizante MAP- Mono-amônio-fosfato (48\% $\left.\mathrm{P}_{2} \mathrm{O}_{5}\right)$; (2)Fonte de Fertilizante $\mathrm{KCl}$ - Cloreto de Potássio $\left(58 \% \mathrm{~K}_{2} \mathrm{O}\right)$; NC: Necessidade de Calagem; QC: Quantidade de calcário; NG: Necessidade de Gesso; QG: Quantidade de gesso; NC=QC (SC:100\%; PF: $20 \mathrm{~cm}$; PRNT:100); NG=QG (SC:100\%; PF: $20 \mathrm{~cm}$ ). Preço dos insumos (t - tonelada): calcário: 45,00 R\$; Gesso: 54,00 R\$; MAP: 1553,00 R\$; KCl: 1465,00 R\$.

A aplicação dos corretivos do solo baseado na variabilidade dos atributos do solo por meio da aplicação a taxa variada promove uma economia de 40,93\% em relação a aplicação pela recomendação média da fertilidade. Quanto a aplicação dos fertilizantes o emprego da agricultura de precisão reduz os custos da aplicação em 20,47\%, quando comparado à recomendação baseada na média da fertilidade do solo. De maneira geral as práticas de agricultura de precisão resultam em maior produção, economia e melhor gerenciamento da propriedade.

A recomendação a taxa variada por meio do mapeamento das áreas com uso de ferramentas geoestatísticas, é o princípio para aumentar a produtividade das culturas e melhorar a eficiência do manejo do solo, além de promover uma economia na quantidade de insumos aplicados. Assim a adoções de práticas que levam a maior precisão na agricultura tornam-se essenciais para a homogeneização da fertilidade, que pode contribuir para a melhoria da produção das culturas implantadas e garantir melhor gerenciamento do sistema de produção.

\section{CONCLUSÕES}

A área apresentou diferentes padrões de variabilidade para os diferentes atributos avaliados.
O conhecimento da variabilidade espacial com o uso de ferramentas geoestatísticas reduz o custo com adubação e correção do solo em relação a adoção de técnicas convencionais que padronizam valores médios dos indicadores da fertilidade do solo por glebas de áreas consideradas homogêneas.

É necessária a adoção de práticas coordenadas pelo zoneamento do manejo da fertilidade do solo de forma a promover a construção da fertilidade nas zonas de menor disponibilidade.

\section{REFERÊNCIAS BIBLIOGRÁFICAS}

BOTTEGA, E. L.; QUEIROZ, D. M.; PINTO, F. A. C.; SOUZA, C. M. A. Variabilidade espacial de atributos do solo em sistema de semeadura direta com rotação de culturas no cerrado brasileiro. Revista Ciência Agronômica, v.44, n. 1, p. 1-9, 2013.

CAMBARDELLA, C. A.; MOORMAN, T. B.; NOVAK, J. M.; PARKIN, T. B.; KARLEN, D. L.; TURCO, R. F.; KONOPKA, A. E. Field-scale variability of soil properties in Central Iowa. Soil Science Society of American Journal, v.58, n.5, p. 1501-1511, 1994.

CARNEIRO, J. S. S.; SANTOS, A. C. M.; FIDELIS, R. R.; SILVA NETO, S. P.; SANTOS, A. C.; SILVA, R. R. Diagnóstico e manejo da variabilidade espacial da 
fertilidade do solo no cerrado do Piauí. Revista de Ciências Agroambientais, v.14, n. 2, p. 10-21, 2016.

DALCHIAVON, F. C.; CARVALHO, M. P.; ANDREOTTI, M.; MONTANARI, R. Variabilidade espacial de atributos da fertilidade de um Latossolo Vermelho Distroférrico sob Sistema Plantio Direto. Revista Ciência Agronômica, v.43, n. 3, p. 453-461, 2012.

DALCHIAVON, F. C.; CARVALHO, M. P.; FREDDI, O. S.; ANDREOTTI, M.; MONTANARI, R. Variabilidade espacial da produtividade do feijoeiro correlacionada com atributos químicos de um Latossolo Vermelho Distroférrico sob sistema de semeadura direta. Bragantia, v.70, n. 4, p. 908-916, 2011a.

DALCHIAVON， F. C.; CARVALHO, M. P.; NOGUEIRA, D. C.; ROMANO, D.; ABRANTES, F. L.; ASSIS, J. T.; OLIVEIRA, M. S. Produtividade da soja e resistência mecânica à penetração do solo sob sistema plantio direto no cerrado brasileiro. Pesquisa Agropecuária Tropical, v.41, n. 1, p. 8-19, 2011 b.

DRUCK, S.; CARVALHO, M. S.; CÂMARA, G.; MONTEIRO, A. M. V. Análise espacial de dados geográficos. Embrapa- Empresa Brasileira de Pesquisa Agropecuária, Brasília. 2004. 209p.

EMBRAPA - Empresa Brasileira de Pesquisa Agropecuária. Manual de métodos de análise de solo. 2. ed. Rio de Janeiro: Embrapa Solos. 2011. 230 p.

EMBRAPA- Empresa Brasileira de Pesquisa Agropecuária. Sistema brasileiro de classificação de solos. 3. ed. Brasília: Centro Nacional de Pesquisa de Solos. 2013. $353 \mathrm{p}$.

LEITE, L. F. C.; FERREIRA, J. S.; VELOSO, M. E. C.; MOUSINHO, F. E. P.; ROCHA JUNIOR, A. F. Variabilidade espacial das frações da matéria orgânica do solo em área degradada sob recuperação. Revista Brasileira de Engenharia Agrícola e Ambiental, v.19, n. 4, p. 394-401, 2015.

LIMA, G. C.; SILVA, M. L. N.; OLIVEIRA, M. S.; CURI, N.; SILVA, M. A.; OLIVEIRA, A. H. Variabilidade de atributos do solo sob pastagens e mata atlântica na escala de microbacia hidrográfia. Revista Brasileira de Engenharia Agrícola e Ambiental, v.18, n. 5, p. 517-526, 2014.

MATIAS, S. S. R.; NÓBREGA, J. C. A.; NÓBREGA, R. S. A.; ANDRADE, F. R.; BAPTISTEL, A. C. Variabilidade espacial de atributos químicos em Latossolo cultivado de modo convencional com soja no cerrado piauiense. Revista Agro@mbiente On-line, v.9, n. 1, p. 17-26, 2015.
MONTANARI, R.; PANACHUKI, E.; LOVERA, L. H.; CORREA, A. R.; OLIVEIRA, I. S.; QUEIROZ, H. A.; TOMAZ, P. K. Variabilidade espacial da produtividade de sorgo e de atributos do solo na região do ecótono Cerrado-Pantanal, MS. Revista Brasileira de Ciência do Solo, v.39, n. 2, p. 385-396, 2015.

NEGREIROS NETO, J. V.; SANTOS, A. C.; GUARNIERI, A.; SOUZA, D. J. A. T.; DARONCH, D. J.; DOT'TO, M. A.; ARAÚJO, A. S. Variabilidade espacial de atributos físico-químicos de um Latossolo Vermelho-Amarelo distrófico em sistema plantio direto. Semina: Ciências Agrárias, v.35, n. 1, p. 193-204, 2014.

RESENDE, J. M. A.; MARQUES JÚNIOR, J.; MARTINS FILHO, M. V.; DANTAS, J. S.; SIQUEIRA, D. S.; TEIXEIRA, D. B. Variabilidade espacial de atributos de solos coesos do leste maranhense. Revista Brasileira de Ciência do Solo, v.38, n. 4, p. 1077-1090, 2014.

RIBEIRO, A. C.; GUIMARÃES, P. T. G.; ALVAREZ, V. V. H. Recomendação para o uso de corretivos e fertilizantes em Minas Gerais: $5^{a}$ aproximação. Viçosa: Comissão de Fertilidade do Solo do Estado de Minas Gerais. 1999. $359 \mathrm{p}$.

SHAPIRO, S. S. \& WILK, M. B. An analyze of variance test for normality: complete samples. Biometrika, v.52, n.3/4, p. 591-611, 1965.

SILVA NETO, S. P.; SANTOS, A. C.; LEITE, R. L. L.; DIM, V. P.; CRUZ, R. S.; PEDRICO, A.; NEVES NETO, D. N. Análise espacial de parâmetros da fertilidade do solo em região de ecótono sob diferentes usos e manejos. Semina: Ciências Agrárias, v.32, n. 2, p. 541-552, 2011a.

SILVA NETO, S. P.; SANTOS, A. C.; SILVA, J. E. C. Variabilidade espacial da fertilidade de neossolo quartzarênico em função da substituição do cerrado por pastagem. Engenharia na Agricultura, v.19, n. 4, p. 334347, 2011b.

SILVA, J. M. Análise espacial de atributos físicos em um latossolo cultivado em plantio direto. Engenharia Ambiental, v.10, n. 3, p. 27 -38, 2013.

WARRICK, A. W. \& NIELSEN, D. R. Spatial variability of soil physical properties in the fild. In: HILLEL, D., (Ed.) Applications of soil physics. New York: Academic Press. 1980. p. 319-344.

ZANÃO JÚNIOR, L. A.; LANA, R. M. Q.; CARVALHO-ZANÃO, M. P.; GUIMARÃES, E. D. Variabilidade espacial de atributos químicos em 
diferentes profundidades em um Latossolo em sistema de plantio direto. Revista Ceres, v.57, n. 3, p. 429-438, 2010 .

ZONTA, J. H.; BRANDÃO, Z. N.; MEDEIROS, J. C.; SANA, R. S.; SOFITTI, V. Variabilidade espacial da fertilidade do solo em área cultivada com algodoeiro no Cerrado do Brasil. Revista Brasileira de Engenharia Agrícola e Ambiental, v.18, n. 6, p. 595-602, 2014. 